\title{
SGLT-2 Inhibitors: Are They a Promising Treatment Option in T2DM Patients with NAFLD?
}

\section{Dimitrios Patoulias*}

\begin{abstract}
Sodium glucose co-transporter type 2 inhibitors (SGLT-2 inhibitors) are a class of antidiabetics, recently approved for the treatment of patients with T2DM. They feature cardioprotective and renoprotective action, while they exert beneficial effects on metabolic parameters. Non-alcoholic fatty liver disease (NAFLD) is a frequent co-morbidity in diabetic patients. Its prevalence reaches up to $70 \%$. Since there is no specific treatment approved for NAFLD, both experimental and clinical studies have been recently conducted highlighting the efficacy and safety of SGLT-2 inhibitors mainly in animal models and secondarily in patients with T2DM and NAFLD. This class of antidiabetics seems very attractive, improving both glycemic control and liver function tests, while inhibiting NAFLD progression. However, further investigation is required to establish them as a first-line treatment option in T2DM patients with NAFLD, after thorough assessment of their efficacy and safety in clinical practice.
\end{abstract}

\section{KEYWORDS}

SGLT-2 inhibitor; NAFLD; T2DM

\section{AUTHOR AFFILIATIONS}

Department of Internal Medicine, General Hospital of Veria, Veria, Greece

* Corresponding author: M. Alexandrou 3B, Pefka, Thessaloniki, Postal code 57010; e-mail: dipatoulias@gmail.com

Received: 8 September 2017

Accepted: 6 February 2018

Published online: 23 April 2018

Acta Medica (Hradec Králové) 2017; 60(4): 167-170

https://doi.org/10.14712/18059694.2018.15

(c) 2017 The Author. This is an open-access article distributed under the terms of the Creative Commons Attribution License (http://creativecommons.org/licenses/by/4.0), which permits unrestricted use, distribution, and reproduction in any medium, provided the original author and source are credited. 


\section{ABBREVIATIONS}

SGLT-2, sodium-glucose co-transporter type 2; DM, diabetes mellitus; T2DM, type 2 diabetes mellitus; NAFLD, non-alcoholic fatty liver disease; NASH, non-alcoholic steatohepatitis; HCC, hepatocellular carcinoma; TC, total cholesterol; FPG, fasting plasma glucose; FFA, free fatty acids

\section{INTRODUCTION}

Non-alcoholic fatty liver disease (NAFLD) is very common among patients with type 2 diabetes mellitus (T2DM). Prevalence of NAFLD in patients with T2DM rises up to $70 \%$, while prevalence of non-alcoholic steatohepatitis (NASH) in T2DM asymptomatic individuals is as high as $20 \%$ (1). As NAFLD can lead to NASH, and the latter to cirrhosis and hepatocellular carcinoma (HCC), physicians should pay extra attention in those cases of concomitance.

SGLT-2 inhibitors are a class of antidiabetic drugs, recently approved for the treatment of patients with T2DM. A few experimental and clinical studies have been conducted aiming at investigating whether SGLT-2 inhibitors can offer as therapeutic option in patients with both T2DM and NAFLD.

Although there is no approved treatment for NAFLD, the presence of a drug class that improves both glycemic control and liver function tests, and inhibits the progression of NAFLD, seems very attractive in clinical practice. Through this brief communication, I aim at presenting both the relevant experimental and clinical data, discussing the potential beneficial effects of SGLT-2 inhibitors on those patients.

\section{PATHOPHYSIOLOGY}

SGLT-2 inhibitors mediated glucosuria leads to decrease in blood glucose levels, and finally to suppression of insulin secretion by pancreatic $\beta$-cells. Glucagon levels are elevated due to the paracrine effect of insulin and the direct effect of SGLT-2 inhibitors on the pancreatic $a$-cells. This hormonal imbalance (increased glucagon/insulin ratio) induces increase in hepatic gluconeogenesis, glucogen breakdown and lipolysis (2).

Daniele et al. documented that dapagliflozin shifts energy metabolism in patients with T2DM from glucose to lipid oxidation. The researchers noticed the presence of increased plasma ketone concentration in patients treated with dapagliflozin. Increased b-oxidation and increased fasting plasma glucagon levels constitute a potential mechanism of increased total ketone levels in those subjects treated with SGLT-2 inhibitors. Ferrannini et al. came to a similar conclusion, with the use of empagliflozin in patients with T2DM $(3,4)$.

In another interesting study by Ferrannini and colleagues in patients with T2DM, it was observed in the acute phase that empagliflozin led to improved glycemia, decrease in tissue glucose disposal and improved $\beta$-cell function and cellular insulin sensitivity, along with enhanced postprandial GLP-1 response. Chronic study con- firmed the shift in substrate utilization from carbohydrate to lipid - as indicated by the increase in FFA concentrations -, with maintenance of the results obtained in the acute phase of the study (5).

Kamei and colleagues showed in their recently published study that tofogliflozin administration in patients with T2DM led significant decrease in body weight, body mass index (BMI), body fat mass and visceral fat area, besides the significant improvement in glycemic control and serum uric acid levels. After univariate analyses, the authors concluded that tofogliflozin seems a more reasonable therapeutic approach in obese individuals with recently diagnosed T2DM, suggesting in fact the phenotype of patients that may benefit most from SGLT-2 inhibitor initiation (6).

Collective and careful interpretation of the above leads to the conclusion that SGLT- 2 inhibitors mediate metabolism shift from glucose to lipid oxidation, enhance $\beta$-cell function, improve cellular glucose sensitivity and lead to significant decrease in body weight, mainly due to reduction in body fat mass and visceral fat area. Thus, it seems reasonable that SGLT-2 inhibitor administration in patients with concomitant T2DM and NAFLD will be much beneficial, with significant improvement in all metabolic parameters.

\section{EXPERIMENTAL DATA}

Honda et al. (7) investigated the effect of ipragliflozin on diet induced obese (DIO) mice, using an amylin liver NASH model (AMLN) for 20 weeks. The researchers found that ipragliflozin reduced levels of total cholesterol (TC), fasting plasma glucose (FPG), insulin, free fatty acids (FFA) and aminotransferase, while it also improved insulin resistance. At pathogenesis level, ipragliflozin improved steatosis grade, decreasing lobular inflammation and lipid droplets deposition, while it improved fibrosis stage in treated mice, as well.

Komiya et al. (8) conducted an experimental study in obese mice with insulin resistance, concluding that ipragliflozin decreased liver weight, hepatic lipid accumulation and triglyceride (TG) deposition, serum ALT levels, along with glucose intolerance, findings supportive of beneficial effects on hepatic steatosis. Downregulation of lipogenic genes and genes involved in macrophages activation explain ipragliflozin effects on hepatic steatosis and inflammation. In a clinical study following the animal study, the authors documented improvement of liver dysfunction in patients with T2DM, without significant weight reduction. They suggest that SGLT-2 inhibitors may serve as optional treatment for hepatic steatosis in T2DM individuals, due to their multifactorial effects on metabolic parameters.

In another DIO mouse model, Nakano et al. (9) documented that after a 4-week course of treatment, remogliflozin decreased serum ALT and AST levels, total liver weight, while it also reduced hepatic inflammation and oxidative stress, as indicated by reduced mRNA expression of TNF-a, MCP-1 and TBARS, respectively. Remogliflozin also improved hepatic steatosis, by reducing TG content in hepatic tissue. 
Tahara et al. (10) investigated the therapeutic impact of ipragliflozin on high-fat diet and streptozotocin-nicotinamide-induced type 2 diabetic mice. The administered SGLT-2 inhibitor improved significantly glycemic control, lipid levels, hepatic lipid content, total body and liver adipose tissue weight and serum levels of AST and ALT. Oxidative stress and inflammatory process were significantly improved, dose-dependently, by ipragliflozin. Thus, ipragliflozin ameliorates hepatic steatosis and progression to $\mathrm{NASH}$, finding indicative of potential use in diabetic patients with NAFLD.

In another experimental study in rats fed on choline-deficient L-amino acid-defined diet, with induced hepatic steatosis and fibrosis, Hayashizaki-Someya et al. (11) documented that ipragliflozin: a) suppressed body weight gain, b) reduced hepatic TG content, c) decreased hepatic fibrosis score in a dose-dependent manner and d) did not affect serum AST and ALT levels. The authors suggest fat utilization as the main factor that led to decrease in steatosis. In accordance with the previous studies was that conducted by Qiang et al. (12). The researchers developed a rodent model with T2DM and NASH and evaluated the efficacy of luseogliflozin on metabolic parameters. In mice treated with the SGLT-2 inhibitor, the authors noticed normalization of serum ALT levels, decrease in hepatic steatosis and improvement in inflammatory and fibrotic changes.

Ji et al. (13) evaluated the beneficial effect of canagliflozin on metabolic parameters performing an experimental study in DIO mice, which did not feature or develop T2DM. It decreased body weight in DIO mice, reduced serum levels of TC and TG, but more significantly, it improved hepatic steatosis, reversing the effects of high fat diet on liver weight and liver to body weight ratio. Up-regulation of PPARal and down-regulation of DGAT2, PPAR $\gamma 1$ and PPAR $\gamma 2$ may explain this effect on hepatic steatosis.

Jojima et al. (14) confirmed the synergistic effect of empagliflozin and linagliptin on inhibition of NASH progression in a mouse model of NASH and DM, however lacking obesity and insulin resistance. The authors found that empagliflozin reduced serum ALT, liver to body weight ratio and hepatic TG content, while it also decreased significantly hepatic inflammation. However, anti-fibrotic effect of the combination was more efficient than empagliflozin or linagliptin alone. Another interesting finding was that the combination significantly reduced FAS and ACC1 mRNA expression, genes both involved in lipogen- esis, while neither empagliflozin nor linagliptin affected expression of ACOX1 and PPAR- $\alpha$, genes involved in $\beta$-oxidation, in contrast with Ji et al.

Obara et al. (15) identified an effect of tofogliflozin on the progression of NAFLD towards cirrhosis and hepatocellular carcinoma (HCC). The researchers administered tofogliflozin in diabetic and obese mice for 2 weeks, while they were also administered diethylnitrosamine-containing water. They concluded that tofogliflozin improved hepatic steatosis and inflammation, reduced serum glucose and FFA levels, while it did not affect insulin resistance. A much more interesting finding was that the administered SGLT-2 inhibitor suppressed the development of hepatic paraneoplastic lesions, due to attenuation of NAFLD progression and not due to direct effect on HCC cells.

The main results of the above discussed experimental studies are summarized on table 1 .

\section{CLINICAL DATA}

There are only a few clinical data regarding the usefulness of SGLT-2 inhibitors in NAFLD until now. In their clinical study in 11 patients with T2DM and NASH, Tobita et al. (16) affirmed that dapagliflozin after 24 weeks of treatment reduced body fat mass and percent body fat, improved liver tests and glycemic parameters. The authors associated: a) lower ferritin levels with improvement in hepatic fibrosis and b) improvement in glycemic control and lower insulin levels with decrease in insulin resistance. Despite the small sample of patients, the short-term study and the absence of liver biopsies, the above results are promising as for the use of SGLT-2 inhibitors in patients with metabolic syndrome. In a recently published randomized controlled trial, patients with T2DM and NAFLD were randomized to receive either ipragliflozin or pioglitazone for 24 weeks. Improvement in NAFLD values and glycemic control were equal between the two groups. However, only ipragliflozin reduced significantly body weight and abdominal fat area, enhancing its usefulness as treatment option in patients with T2DM and NAFLD (17).

In a pooled analysis, Leiter et al. (18) documented that canagliflozin was superior to placebo or sitagliptin regarding the improvement of liver function tests in individuals with T2DM, finding consistent with substantial improvement in glycemic parameters and reduction in total body weight. Finally, Okhi et al. (19) administered

Tab. 1 Effect of SGLT-2 inhibitors on hepatic steatosis, hepatic fibrosis and tumorigenesis (experimental models).

\begin{tabular}{|l|l|l|l|}
\hline & Administered SGLT-2 inhibitor & Hepatic steatosis & Hepatic fibrosis \\
\hline Honda et al (7) & Ipragliflozin & $\checkmark$ & $\checkmark$ \\
\hline Komiya et al (8) & Ipragliflozin & $\checkmark$ & $\checkmark$ \\
\hline Nakano et al (9) & Remogliflozin & $\checkmark$ & \\
\hline Tahara et al (10) & Ipragliflozin & $\checkmark$ & $\checkmark$ \\
\hline Hayashizaki-Someya et al. (11) & Ipragliflozin & & \\
\hline Qiang et al. (12) & Luseogliflozin & $\checkmark$ & \\
\hline Ji et al. (13) & Canagliflozin & $\checkmark$ & \\
\hline Jojima et al. (14) & Empagliflozin & $\checkmark$ & $\checkmark$ \\
\hline Obara et al. (15) & Tofogliflozin & $\checkmark$ & \\
\hline
\end{tabular}


an SGLT-2 inhibitor in 24 patients with T2DM and NAFLD previously receiving either a DPP-4 inhibitor or a GLP-1 analog. After 320 days of administration, the researchers observed not only a further improvement in glycemic control, but also decrease in serum ALT levels and body weight and improvement in FIB-4 index. SGLT-2 inhibitors seem an interesting alternative in patients with NAFLD and T2DM, who did not respond to incretin-based therapies.

\section{CONCLUSION}

Based upon the above mentioned, primarily, experimental and, secondly, clinical data, although it is early to reach a safe conclusion, SGLT-2 inhibitors share some certain characteristics: they act against inflammation and oxidative stress, decrease hepatic steatosis, prevent the progression towards hepatic fibrosis or even reverse fibrotic changes in liver parenchyma and they possibly act against tumorigenesis. According to available data, they seem superior to other antidiabetics, and they can act synergistically, as well. Further investigation regarding their efficacy and safety in well-designed randomized controlled trials in humans will clarify whether they can be established as first-line therapeutic option or not.

I consider that this class of antidiabetics constitutes a very attractive therapeutic option in patients with T2DM and NAFLD.

\section{CONFLICT OF INTEREST}

The author declares that he does not have any conflict of interest, including specific financial interests or relationships and affiliations relevant to the subject matter or materials discussed in the manuscript.

\section{REFERENCES}

1. Hazlehurst JM, Woods C, Marjot T, Cobbold JF, Tomlinson JW. Non-alcoholic fatty liver disease and diabetes, Metabolism 2016, 65(8): 1096-1108.

2. Taylor S, Blau J, Rother, K. SGLT2 Inhibitors May Predispose to Ketoacidosis. J. Clin. Endocrinol Metab 2015; 100(8): 2849-2852.

3. Daniele G, Xiong J, Solis-Herrera C, et al. Dapagliflozin Enhances Fat Oxidation and Ketone Production in Patients With Type 2 Diabetes. Diabetes Care 2016; 39(11): 2036-2041.

4. Ferrannini E, Baldi S, Frascerra S, et al. Shift to fatty substrate utilization in response to sodium-glucose cotransporter 2 inhibition in subjects without diabetes and patients with type 2 diabetes. Diabetes 2016; 65: 1190-1195.

5. Ferrannini E, Muscelli E, Frascerra S, et al. Metabolic response to sodium-glucose cotransporter 2 inhibition in type 2 diabetic patients. J Clin Invest 2014; 124(2): 499-508.

6. Kamei S, Iwamoto M, Kameyama M, et al. Effect of Tofogliflozin on Body Composition and Glycemic Control in Japanese Subjects with Type 2 Diabetes Mellitus. Journal of Diabetes Research 2018, Article ID 6470137, 6 pages.

7. Honda Y, Imajo K, Kato T, Kessoku T, Ogawa Y, Tomeno W, et al. The Selective SGLT2 Inhibitor Ipragliflozin Has a Therapeutic Effect on Nonalcoholic Steatohepatitis in Mice. PLoS ONE 2016; 11(1): e0146337.

8. Komiya C, Tsuchiya K, Shiba K, Miyachi Y, Furuke S, Shimazu N, et al. Ipragliflozin Improves Hepatic Steatosis in Obese Mice and Liver Dysfunction in Type 2 Diabetic Patients Irrespective of Body Weight Reduction. PLoS ONE 2016; 11(3): e0151511.

9. Nakano S, Katsuno K, Isaji M, et al. Remogliflozin Etabonate Improves Fatty Liver Disease in Diet-Induced Obese Male Mice. J Clin Exp Hepatol 2015; 5(3): 190-198.

10. Tahara A, Kurosaki E, Yokono M, et al. Effects of SGLT2 selective inhibitor ipragliflozin on hyperglycemia, hyperlipidemia, hepatic steatosis, oxidative stress, inflammation, and obesity in type 2 diabetic mice. Eur J Pharmacol 2013; 715(1-3): 246-255.

11. Hayashizaki-Someya Y, Kurosaki E, Takasu T, et al. Ipragliflozin, an SGLT2 inhibitor, exhibits a prophylactic effect on hepatic steatosis and fibrosis induced by choline-deficient l-amino acid-defined diet in rats. Eur J Pharmacol 2015; 754: 19-24.

12. Qiang S, Nakatsu Y, Seno Y, et al. Treatment with the SGLT2 inhibitor luseogliflozin improves nonalcoholic steatohepatitis in a rodent model with diabetes mellitus. Diabetol Metab Syndr 2015; 7: 104.

13. Ji W, Zhao M, Wang M, Yan W, Liu Y, Ren S, et al. Effects of canagliflozin on weight loss in high-fat diet-induced obese mice. PLoS ONE 2017; 12 (6): e0179960.

14. Jojima T, Tomotsune T, Iijima T, Akimoto K, Suzuki K, Aso Y. Empagliflozin (an SGLT2 inhibitor), alone or in combination with linagliptin (a DPP-4 inhibitor), prevents steatohepatitis in a novel mouse model of non-alcoholic steatohepatitis and diabetes. Diabetol Metab Syndr 2016; 8: 45.

15. Obara K, Shirakami Y, Maruta A, et al. Preventive effects of the sodium glucose cotransporter 2 inhibitor tofogliflozin on diethylnitrosamine-induced liver tumorigenesis in obese and diabetic mice. Oncotarget 2017; 8(35): 58353-58363.

16. Tobita H, Sato S, Miyake T, Ishihara S, Kinoshita Y. Effects of dapagliflozin on body composition and liver tests in patients with non-alcoholic steatohepatitis associated with type 2 diabetes mellitus: A prospective, open-label, uncontrolled study. Curr Ther Res Clin Exp 2017; 87: 13-19.

17. Ito D, Shimizu S, Inoue K, et al. Comparison of Ipragliflozin and Pioglitazone Effects on Nonalcoholic Fatty Liver Disease in Patients With Type 2 Diabetes: A Randomized, 24-Week, Open-Label, Active-Controlled Trial. Diabetes Care 2017; 40(10): 1364-1372.

18. Leiter LA, Forst T, Polidori D, Balis DA, Xie J, Sha S. Effect of canagliflozin on liver function tests in patients with type 2 diabetes, Diabetes Metab 2016; 42(1): 25-32.

19. Okhi T, Isogawa A, Toda N, Tagawa K. Effectiveness of Ipragliflozin, a Sodium-Glucose Co-transporter 2 Inhibitor, as a Second-line Treatment for Non-Alcoholic Fatty Liver Disease Patients with Type 2 Diabetes Mellitus Who Do Not Respond to Incretin-Based Therapies Including Glucagon-like Peptide-1 Analogs and Dipeptidyl Peptidase-4 Inhibitors. Clin Drug Investig 2016; 36(4): 313-319. 